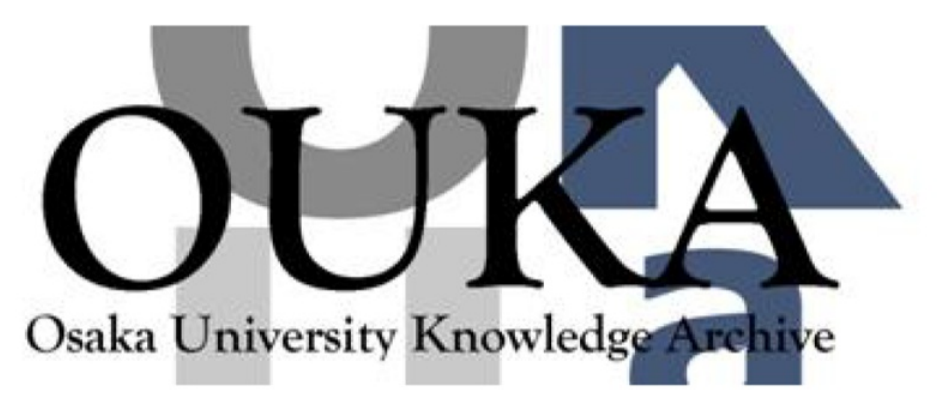

\begin{tabular}{|c|l|}
\hline Title & $\begin{array}{l}\text { Electric field tuning of a stop band in a } \\
\text { reflection spectrum of synthetic opal } \\
\text { infiltrated with nematic liquid crystal }\end{array}$ \\
\hline Author(s) & Shimoda, Y.; Ozaki, Masanori; Yoshino, Katsumi \\
\hline Citation & Applied Physics Letters. 79(22) p. 3627-p. 3629 \\
\hline Issue Date & $2001-11-16$ \\
\hline oaire:version & VoR \\
\hline URL & https://hdl. handle. net/11094/75835 \\
\hline rights & \\
\hline Note & \\
\hline
\end{tabular}

Osaka University Knowledge Archive : OUKA

https://ir. Library. osaka-u. ac. jp/

Osaka University 


\section{Electric field tuning of a stop band in a reflection spectrum of synthetic opal infiltrated with nematic liquid crystal}

Cite as: Appl. Phys. Lett. 79, 3627 (2001); https://doi.org/10.1063/1.1421080

Submitted: 08 February 2001 . Accepted: 19 September 2001 . Published Online: 16 November 2001

Y. Shimoda, M. Ozaki, and K. Yoshino

\section{ARTICLES YOU MAY BE INTERESTED IN}

Temperature tuning of the stop band in transmission spectra of liquid-crystal infiltrated synthetic opal as tunable photonic crystal

Applied Physics Letters 75, 932 (1999); https://doi.org/10.1063/1.124558

Temperature effect on liquid crystal refractive indices

Journal of Applied Physics 96, 19 (2004); https://doi.org/10.1063/1.1757034

VOLTAGE-DEPENDENT OPTICAL ACTIVITY OF A TWISTED NEMATIC LIQUID CRYSTAL Applied Physics Letters 18, 127 (1971); https://doi.org/10.1063/1.1653593

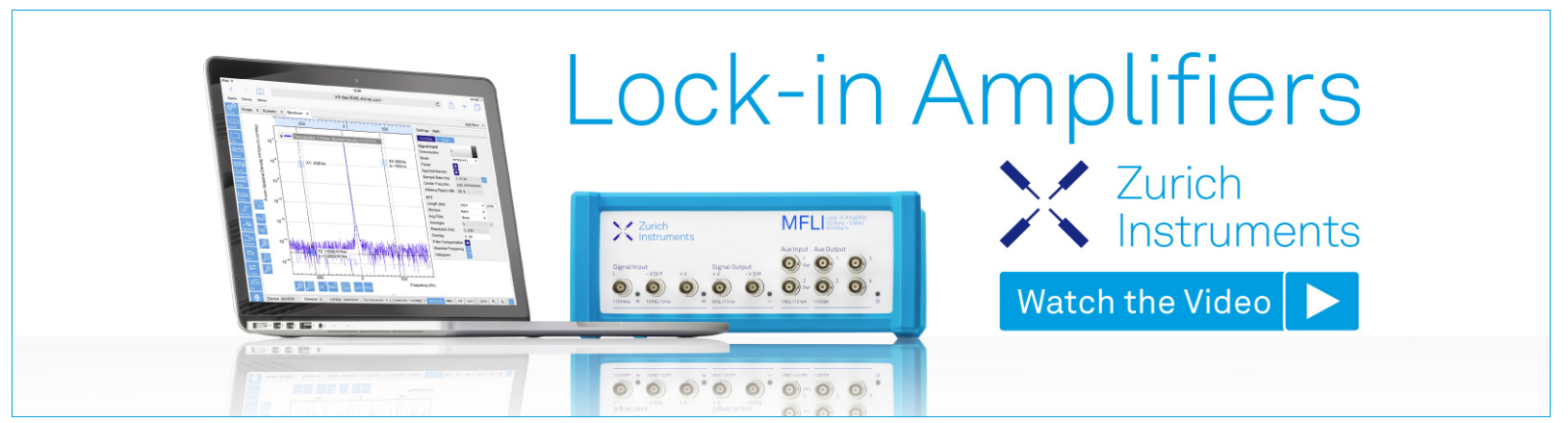




\title{
Electric field tuning of a stop band in a reflection spectrum of synthetic opal infiltrated with nematic liquid crystal
}

\author{
Y. Shimoda, M. Ozaki, ${ }^{\text {a) }}$ and K. Yoshino \\ Department of Electronic Engineering, Graduate School of Engineering, Osaka University, \\ 2-1 Yamada-Oka, Suita, Osaka 565-0871, Japan
}

(Received 8 February 2001; accepted for publication 19 September 2001)

\begin{abstract}
Electric field tuning of an optical stop band in a reflection spectrum is demonstrated in a synthetic opal thin film infiltrated with liquid crystal. The reflection peak of the opal shifts upon the application of the electric field, and this is based on the refractive index change caused by molecular reorientation upon application of a field. The dynamic response of the stop band shift was also studied, and fast response of the order of microsecond was observed. (C) 2001 American Institute of Physics. [DOI: 10.1063/1.1421080]
\end{abstract}

Recently photonic crystals with a three-dimensionally ordered structure with periodicity of the optical wavelength have attracted much attention from both fundamental and practical points of view, because physical concepts such as photonic band gaps have been theoretically predicted and various applications of photonic crystals have been proposed. ${ }^{1,2}$ Synthetic opals have been studied as pseudophotonic crystals or prototype photonic crystals to establish the growth techniques and to understand fundamental optical properties such as the stop band of the three-dimensional periodic structure of materials. ${ }^{3-5}$

We have already demonstrated that various materials can be infiltrated into interconnected nanosize voids of opals, and novel functionalities can be realized in these infiltrated opals. ${ }^{3,6-8}$ Based on this fabrication technique, the concept of a tunable photonic crystal is proposed, in which the photonic band gap can be tuned as desired by controlling parameters such as the refractive index, periodicity or space filling factor. We also propose an anisotropic photonic crystal, in which a three-dimensional periodic array of interconnected voids is infiltrated with anisotropic materials such as liquid crystals. ${ }^{9,10}$

Liquid crystal (LC) has a large optical anisotropy due to its anisotropic molecular shape and alignment, and its alignment is extremely sensitive to external parameters such as the temperature and electric field. As a result, the refractive index of a LC can be modulated by a change in temperature and/or application of an electric field. Based on these characteristics, the optical properties of a synthetic opal whose interconnected nanoscale voids are filled with LCs should easily be controlled by the temperature and electric field. ${ }^{11-14}$

So far, we have already demonstrated a stop band shift upon a change in temperature in a synthetic opal infiltrated with nematic and smectic LCs. ${ }^{11,12}$ In this study we demonstrate tuning of the stop band upon the application of the electric field in a synthetic opal infiltrated with nematic LCs.

Ordered colloidal crystal in the film was formed by sedimentation of the suspension of monodispersed silica spheres $300 \mathrm{~nm}$ in diameter in a sandwich cell made of two indiumtin oxide (ITO) coated glass plates separated by $12 \mu \mathrm{m}$. Po-

a)Electronic mail: ozaki@ele.eng.osaka-u.ac.jp rous opals prepared by this procedure have a face-centeredcubic (fcc) structure and contain an interconnected structure of tetrahedral and octahedral voids. A nematic LC, 4-pentyl4 ' -cyanobiphenyl (5CB, Merck), was used for infiltration.

Reflection characteristics of the opal films were measured with a spectrometer (UV-3100PC, Shimadzu). For measurement of the dynamic optical response to the electric field, the light of a semiconductor laser $(\lambda=680 \mathrm{~nm})$ transmitted through the opal film was detected with a photodiode.

Figure 1 shows reflection peaks of the opal infiltrated with $5 \mathrm{CB}$ as a function of the amplitude of the applied rectangular voltage $(f=1 \mathrm{kHz})$. The light was irradiated perpendicular to the opal film. As is evident in Fig. 1, the reflection peak shifts toward shorter wavelengths (blueshifts) with an increase in voltage. This shift originates from the change in refractive index caused by molecular reorientation along the applied electric field. In the initial state, the refractive index of the LC in nanosize voids of the opal should be macroscopically averaged and it is equivalent to that in the isotropic phase, because of the three-dimensional symmetry of the arrangement and the shape of the voids without an electric field. Under an applied electric field, however, the LC molecules align along the field parallel to the direction of light propagation, and the ratio of the molecules aligned parallel to light propagation slightly increases. As a result, the component of the refractive index $n_{o}$ for ordinary light increases in each void, and the average refractive index of the LC

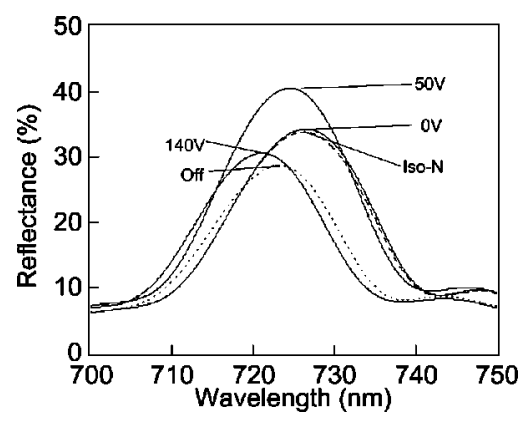

FIG. 1. Reflection spectra of the opal infiltrated with $5 \mathrm{CB}$ as a function of the applied voltage. The reflection peaks after removing the electrical field (off) and after subsequently heating up to the isotropic phase (iso- $N$ ) are also presented. 


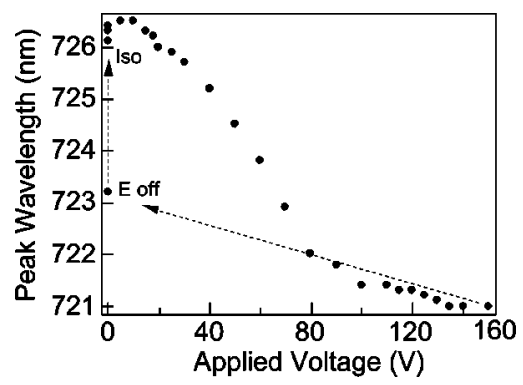

FIG. 2. Applied voltage dependence of the reflection peak wavelength of the opal infiltrated with $5 \mathrm{CB}$. The peak wavelength after turning off the electric field ( $E$ off) and after subsequently heating up to the isotropic phase (iso) are also shown.

decreases. Consequently, the reflection peak shifts toward shorter wavelength.

Figure 2 shows the voltage dependence of the reflection peak wavelength of the opal infiltrated with $5 \mathrm{CB}$. As is evident from Fig. 2, the reflection peak shifts toward shorter wavelength above $10 \mathrm{~V}$ with an increase in applied voltage. The wavelength shift of the reflection peak upon the application of $160 \mathrm{~V}$ is about $5.5 \mathrm{~nm}$, which corresponds to a change in effective refractive index of 0.04 for the infiltrated LC. The refractive indices of $5 \mathrm{CB}$ for ordinary and extraordinary light $720 \mathrm{~nm}$ in wavelength, $n_{o}$ and $n_{e}$, are 1.522 and 1.706, respectively. ${ }^{15}$ Assuming random orientation of the LC molecules in the voids, the averaged refractive index $n_{\text {ave }}$ is calculated to be 1.584 . If all molecules in the voids are completely oriented toward the electric field, the effective refractive index should change by $0.06\left(=n_{\mathrm{ave}}-n_{o}\right)$.

In general, the LC molecules near the solid surface are reoriented very little by the applied electric field. If the volume of the voids infiltrated with liquid crystals is large, the ratio of the region in which the molecules are bounded by the surface is negligible with respect to the total volume. However, in the case of the small voids of the synthetic opal used in this study, the volume of each void is about $0.005 \mu \mathrm{m}^{3}$ and the restricted region in the total volume of the void cannot be neglected. Therefore, the effective refractive index under the field should be larger than $n_{o}$ corresponding to complete alignment of the LC molecules along the electric field. That is why the field-induced change in refractive index of LCs in the opal calculated from the reflection peak shift (0.04) is slightly smaller than that estimated based on the assumption of complete LC molecule alignment to the field (0.06).

It should be also noted that the nematic LC infiltrated into the opal exhibits an optical memory effect. That is, once the reflection peak shifts toward a shorter wavelength, it does not revert back to that in the initial state even after the removal of applied voltage. This may originate from a strong anchoring effect in the small voids of the opal. However, the memory effect disappears when the isotropic phase is heated.

Figure 3 shows typical electrooptic responses of $5 \mathrm{CB}$ infiltrated opal to the applied voltage of a bipolar rectangular wave form $(f=10 \mathrm{kHz})$ of $140 \mathrm{~V}$ in amplitude at $32^{\circ} \mathrm{C}$. As can be seen, the response time is shorter than that of a conventional twisted nematic (TN) cell. In particular, the rise time (dark to bright) shown in Fig. 3(a) is of $\mu$ s order, which is comparable to that in a ferroelectric liquid crystal. The
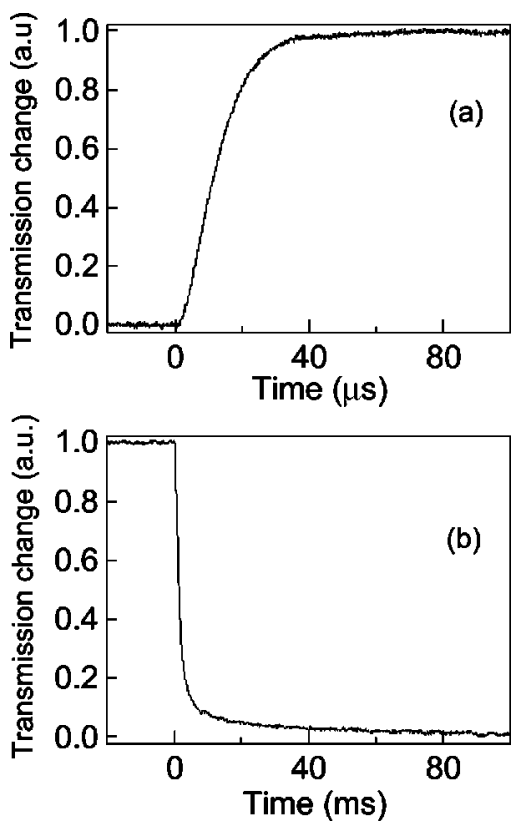

FIG. 3. Optical responses of 5CB infiltrated opal for the application (a) and removal (b) of bipolar rectangular voltage of $140 \mathrm{~V}(f=10 \mathrm{kHz})$ at $32{ }^{\circ} \mathrm{C}$. The light was irradiated normal to the opal plate and the light intensity transmitted was monitored.

origin of the fast response of the nematic LC molecules is not clear at this stage but may be attributed to the deformed nonspherical shape of the voids. In addition, this nonspherical shape of voids with sharp corners that induce defects in the LC alignment might enhance the electric field with respect to the applied field.

Figure 4 shows the voltage dependence of the rise time at 27 and $32^{\circ} \mathrm{C}$. The rise time is defined as the time required to change the transmission intensity from $10 \%$ to $90 \%$ of its total change when bipolar rectangular voltage of $10 \mathrm{kHz}$ is applied. From Fig. 4, it was confirmed that the gradient of the voltage versus the rise time slope is about -2 . That is, the rise time is inversely proportional to the square of the applied voltage. This relationship suggests that the optical response of LC molecules in the voids originates from the dielectric anisotropy. The rise response also depends on the temperature and the response time at $27^{\circ} \mathrm{C}$ is longer than that at $32{ }^{\circ} \mathrm{C}$, which must be associated with the temperature dependence of the viscosity of $5 \mathrm{CB}$.

In summary, we demonstrated that the position of the stop band in the opal infiltrated with a nematic liquid crystal

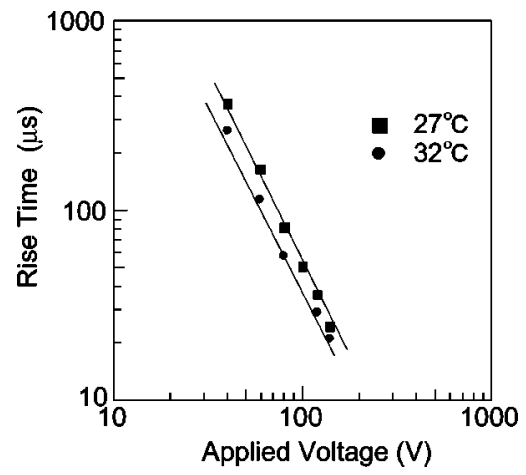

FIG. 4. Applied voltage dependence of the response time to application of the electric field (rise time) at 27 and $32{ }^{\circ} \mathrm{C}$. 
could be tuned by applying voltage, which should confirm the possibility of a tunable photonic crystal. It was also found that the response of a nematic LC in nanosize voids of opal was much faster than that of a conventional electrooptic device based on a nematic LC.

This work was supported by a Grant-in-Aid for Scientific Research from the Japan Ministry of Education, Culture, Sports, Science and Technology.

${ }^{1}$ S. John, Phys. Rev. Lett. 58, 2486 (1987).

${ }^{2}$ E. Yablonovitch, Phys. Rev. Lett. 58, 2059 (1987).

${ }^{3}$ K. Yoshino, K. Tada, M. Ozaki, A. A. Zakhidov, and R. H. Baughman, Jpn. J. Appl. Phys., Part 2 36, L714 (1997).

${ }^{4}$ Y. A. Vlasov, V. N. Astratov, O. Z. Karimov, and A. A. Kaplyanskii, Phys. Rev. B 55, 13357 (1997).

${ }^{5}$ H. Miguez, C. Lopez, F. Meseguer, A. Blanco, L. Vazquez, R. Mayoral,
M. Ozana, V. Fornes, and A. Mifsud, Appl. Phys. Lett. 71, 1148 (1997).

${ }^{6}$ K. Yoshino, S. B. Lee, S. Tatsuhara, Y. Kawagishi, M. Ozaki, and A. A. Zakhidov, Appl. Phys. Lett. 73, 3506 (1998).

${ }^{7}$ K. Yoshino, S. Tatsuhara, Y. Kawagishi, M. Ozaki, A. A. Zakhidov, and Z. V. Vardeny, Jpn. J. Appl. Phys., Part 2 37, L1187 (1998).

${ }^{8}$ K. Yoshino, S. Tatsuhara, Y. Kawagishi, M. Ozaki, A. A. Zakhidov, and Z. V. Vardeny, Appl. Phys. Lett. 74, 2590 (1999).

${ }^{9}$ K. Yoshino, Prod. Tech. 50, 26 (1998) (in Japanese).

${ }^{10}$ K. Busch and S. John, Phys. Rev. Lett. 83, 967 (1999).

${ }^{11}$ K. Yoshino, Y. Shimoda, Y. Kawagishi, K. Nakayama, and M. Ozaki, Appl. Phys. Lett. 75, 932 (1999).

${ }^{12}$ K. Yoshino, S. Satoh, Y. Shimoda, Y. Kawagishi, K. Nakayama, and M. Ozaki, Jpn. J. Appl. Phys., Part 2 38, L961 (1999).

${ }^{13}$ S. W. Leonard, J. P. Mondia, H. M. van Driel, O. Toader, S. John, K. Busch, A. Birner, U. Gösele, and V. Lehmann, Phys. Rev. B 61, 2389 (2000).

${ }^{14}$ D. Kang, J. E. Maclennan, N. A. Clark, A. A. Zakhidov, and R. H. Baughman, Phys. Rev. Lett. 86, 4052 (2001).

${ }^{15}$ S.-T. Wu, J. Appl. Phys. 69, 2080 (1991). 